\title{
Sufficient Authentication for Energy Consumption in Wireless Sensor Networks
}

\author{
Riaz Shaik*, Lokesh Kanagala*, Hema Gopinath Sukavasi * \\ * Department of Computer Science and Engineering, KL University, India
}

\begin{tabular}{l} 
Article Info \\
\hline Article history: \\
Received Sep 15, 2015 \\
Revised Nov 22, 2015 \\
Accepted Dec 13, 2015 \\
\hline Keyword: \\
Energy conservation \\
Key management \\
Network scalability \\
Reliability \\
Secure connectivity coverage \\
Security \\
Wireless sensor networks
\end{tabular}

Wireless sensor networks

\begin{abstract}
Given the understanding of the prospective WSN programs and because of source restrictions, key management emerges as a complicated problem for WSNs. One of the main issues when developing a key management scheme is the system scalability. Indeed, the method should assistance a huge number of nodes to allow a large range implementation of the system. In this paper we implemented a performance trade-off research of power intake vs. Quality of Solutions obtain in stability, suitability, and security for redundancy control of clustered heterogeneous wireless indicator systems using multipath routing to response customer concerns. We urbanized a novel probability style to evaluate the best redundancy stage in terms of direction redundancy $\left(\mathrm{m}_{\mathrm{p}}\right)$ and resource redundancy $\left(\mathrm{m}_{\mathrm{s}}\right)$, as well as the best attack identification configurations with regards to the number of voters $(\mathrm{m})$ and the attack incantation interval under which the life-time of a heterogeneous wireless sensor network is optimized while fulfilling the stability, timeliness and protection specifications of question processing applications in the existence of untrustworthy wireless communication and harmful nodes. Lastly, we used our analysis outcomes to the style of powerful redundancy management criteria to recognize and implement the best design parameter configurations at playback in reaction to environment changes to extend the program life-time.
\end{abstract}

Copyright (C) 2016 Institute of Advanced Engineering and Science. All rights reserved.

\section{Corresponding Author:}

Hema Gopinath Sukavasi

Department of Computer Science and Engineering, KL University

Green fields, Vaddeswaram, Guntur District, Andhra Pradesh, India

Mobile: +91-8500491897

Email: sukavasigopi@gmail.com

\section{INTRODUCTION}

Nowadays, Wireless indicator systems (WSNs) are progressively used in crucial programs within several areas such as army, medical and commercial areas. Key management is an area rock for many security services such as privacy and verification which are required to protected emails in WSNs, the organization of protected hyperlinks between nodes is then a complicated problem in WSNs [1]. Because of source restrictions, symmetrical key organization is one of the most appropriate paradigms for obtaining transactions in WSNs. On the other hand, because of the lack of facilities in WSNs, we have usually no reliable third party which can feature pair wise key important factors to nearby nodes, that is why most current alternatives are depending on key pre-distribution. Over the last several years, a variety of study handled symmetrical key pre-distribution problem for WSNs and many alternatives have been suggested in the literary works [2]. Nevertheless, in most current alternatives, the style of key jewelry (blocks of keys) is highly relevant to the system size, these alternatives either experience from low scalability (number of reinforced nodes), or break down other efficiency analytics such as protected connection, storage space expense and resiliency in the case of huge systems. In this perform; our aim is to deal with the scalability problem without degrading the other system efficiency analytics [3]. For this purpose, we focus on the style 
of a plan which guarantees an excellent secure coverage of extensive systems with a low key storage space expense and an excellent system resiliency. To this end, we make use, for the first time, of the unital style concept for efficient WSN key pre-distribution [1]. Indeed, we recommend an innocent applying from unital style to key pre-distribution and we show through systematic research that it allows achieving a very great scalability [4]. However, this innocent applying does not assurance a higher key discussing possibility. Therefore, we recommend an improved unital-based key pre-distribution plan that preserves an excellent key discussing possibility while improving the system scalability [3].

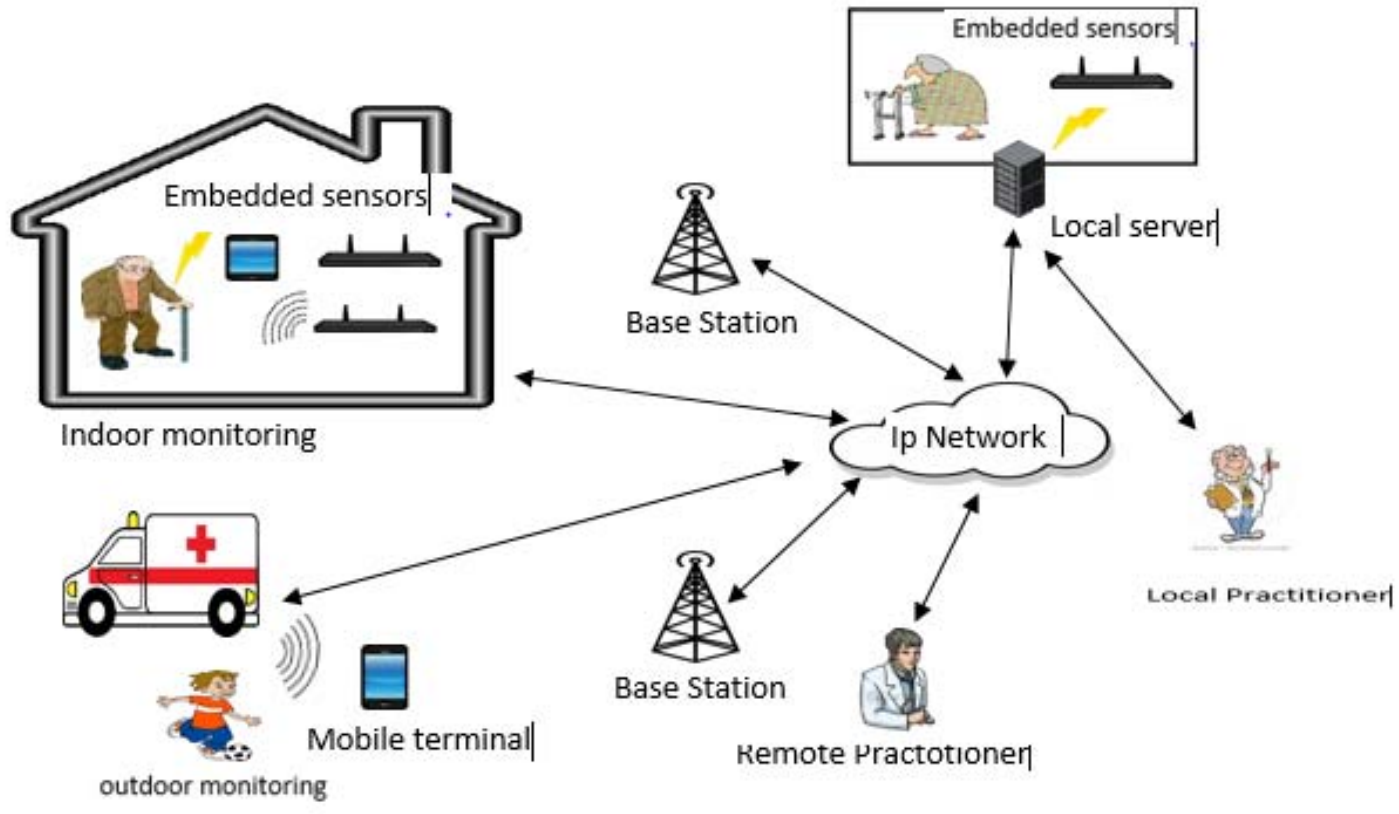

Figure 1. Wireless sensor network Architecture

With the aim of decreasing energy intake while taking the algorithmic complexness into consideration, we recommend a novel strategy that divides the unique information into several packets such that each node in the program will forward only little sub packages. The breaking process is achieved applying the China Rest Theorem (CRT) criteria, which is recognized by an easy flip division between integers [4]. The drain node, once all sub packets (called CRT components) are obtained properly, will recombine them, thus rebuilding the unique concept. The breaking process is especially beneficial for those forwarding nodes that are more seek than others due to their place within the program. Regarding the complexness, in the suggested strategy, almost all nodes function as in a classical sending criterion and, with the exemption of the drain, a few low-complex mathematics functions are needed. 


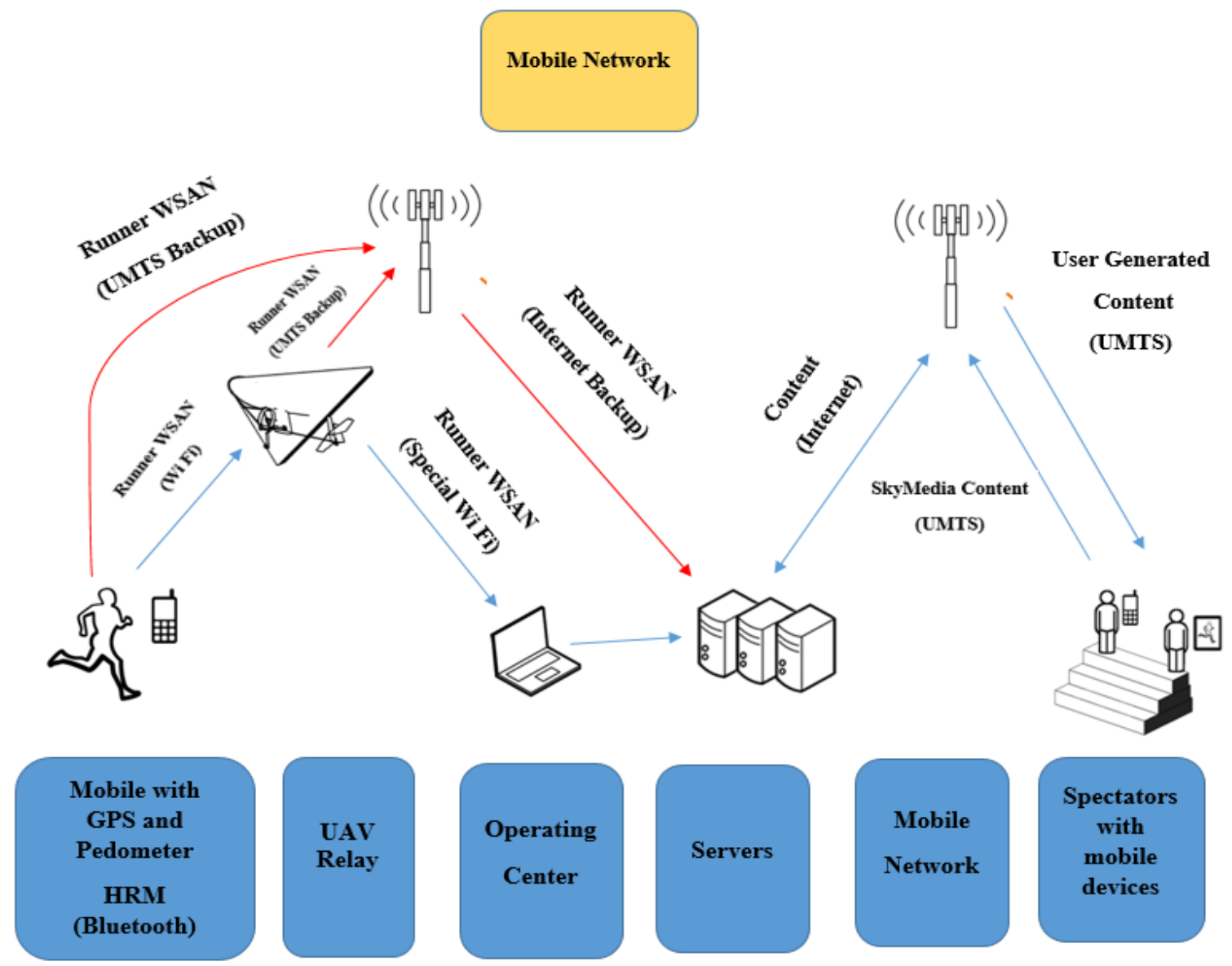

Figure 2. Energy conversion events in wireless sensor networks

More particularly, we analyze the optimal amount of redundancy through which information are directed to a remote drain in the existence of untrustworthy and malicious nodes, so that the question achievements possibility is maximized while improving the HWSN life-time [5]. But some obstacles interrupt the systems performance differently such as the improving bundle wait, thus difficult for reordering the packages, Marketing is not effectively handled also Streaming issue in low data transfer usage information thus reduces performance. To be able to get over the disadvantages of the formerly suggested program, we apply the new idea in this document. In our suggested program, the best possible get in touch with range and interaction technique were mixture to implement the Heterogeneous Wireless Sensor Networks lifestyle in nature [3]. In HWSN, the intra-cluster arranging and inter cluster multi-hop redirecting offer to take advantage the network lifetime. And it is regarded as a hierarchal HWSN with $\mathrm{CH}$ nodes such as excellent energy and providing out capabilities than regular SNs [4]. Our suggested strategy gives solution to come up with as optimization problems to balance energy intake across all nodes in the entire heterogeneous sensor systems [6]. Though in this document, we suggest two-tier HWSN with the objective of capitalizing on program life-time while satisfying energy control and coverage goals. They identified the maximum density ratio of the two tier's nodes to increase the program life-time [6].

The relax of the document will be organized as follows: In section 2, we see about the relevant performs of the document. In section 3, we talk about the suggested technique. The algorithms and simulator are proven in the area 4 and 5.

\section{RELATED WORK}

We research a design of a wireless ad hoc network where nodes match in irrelevant to the resourcetarget sets [7]. These wireless nodes are tacit to be mobile for the interaction systems. The perspective concept offers with the lifestyle and development of techniques of limited places whose crossing points have specified mathematical qualities. Officially, A t-design (, b, r, k, )defined as follows : Given a limited set X of factors (elements), we build a group of b subsets of X, known as prevents, such that each prevent has a

Sufficient Authentication for Energy Consumption in Wireless Sensor Networks (Hema Gopinath Sukavasi) 
dimension $\mathrm{k}$, each factor is included in $\mathrm{r}$ prevents and each $\mathrm{t}$ factors are included together in exactly prevents. For example, the symmetrical Healthy Imperfect Block Design (SBIBD) provided above is a (b, $\mathrm{r}$, $\mathrm{k}$,) style, where $\mathrm{b}=\mathrm{m} 2+\mathrm{m}+1, \mathrm{r}=\mathrm{k}=\mathrm{m}+1$ and $=1$. Typically key submission may not relies on key submission residence, in this we split different prevents. Each prevent contains $m+1$ factors and each factor is included in $r=m 2$ prevents. Each couple of factors is included in exactly one prevent together. We signify the Unital by $2-$ design $(\mathrm{m} 3+1, \mathrm{~m} 2(\mathrm{~m} 2-\mathrm{m}+1), \mathrm{m} 2, \mathrm{~m}+1,1)$ or by $(\mathrm{m} 3+1, \mathrm{~m}+1,1)$ style for convenience benefit [1].

1) Storage space overhead: When using the suggested innocent unital centered edition related a unital of purchase $\mathrm{m}$, each node is pre-loaded with one key band corresponding to one prevent from the style, hence, each node is pre-loaded with $(\mathrm{m}+1)$ disjoint important factors. The storage space needed to shop important factors is then $1 \times(\mathrm{m}+1)$ where 1 is the key dimension.

2) System Scalability: From development, the count of possible key jewelry when using the innocent unital centered plan is $\mathrm{n}=\mathrm{m} 2 \times(\mathrm{m} 3+1)(\mathrm{m}+1)=\mathrm{m} 2 \times(\mathrm{m} 2-\mathrm{m}+1)$, this is then the most of reinforced nodes.

3) Immediate Protected Connection Coverage: When using the primary unital applying, we know that each key is used in exactly $\mathrm{m} 2$ key jewelry among the $\mathrm{m} 2 \times(\mathrm{m} 2-\mathrm{m}+1)$ possible key jewelry. Let us consider two nodes $u$ and $v$ arbitrarily chosen. The node $u$ is pre-loaded with a key band KRu of $m+1$ different important factors. Each of them is included in $\mathrm{m} 2-1$ other key jewelry among the possible $\mathrm{m} 2$ $\times(\mathrm{m} 2-\mathrm{m}+1)-1$ ones. Understanding that two couple of important factors happens together in exactly one prevent, we discover that prevents containing two different important factors of KRu are absolutely disjoint. Hence, each node stocks exactly one key with $(\mathrm{m}+1) \times(\mathrm{m} 2-1)$ nodes among the $\mathrm{m} 2(\mathrm{~m} 2-\mathrm{m}+$ $1)-1$ other possible nodes, then the possibility pc of discussing a typical key can be measured as follows:

$$
P c=\frac{(m+1) x\left(m^{2}-1\right)}{m 2(m 2-m+1)-1}
$$

The assessment of this innocent remedy reveals clearly that the primary applying from unitals to key pre-distribution gives a higher system scalability which gets to $\mathrm{O}(\mathrm{k} 4)$. Moreover, given a system dimension $\mathrm{n}$, this innocent plan allows to decrease the key band dimension up to $\mathrm{p} 4 \mathrm{n}$. However, this innocent remedy outcomes a low key discussing possibility which tends to $\mathrm{O}(1 \mathrm{k})$. To be able to enhance the key discussing possibility while keeping an excellent scalability enhancement, we recommend in the next area an improved scalable and effective unital-based key pre-distribution for WSNs.

\section{BACKGROUND APPROACH}

A new unital-based key pre-distribution plan for WSNs. To be able to enhance the key discussing possibility while keeping great system scalability, we recommend to build the unital style prevents and preload each node with a variety of prevents selected in a particular way [1].

Key Pre-distribution: Before the implementation phase, we produce prevents of $\mathrm{m}$ purchase unital style, where each block corresponds to a key set. We pre-load then each node with $t$ absolutely disjoint prevents where $t$ is a method parameter that we will talk about later in this area. In lemma 1, we illustrate the condition of lifestyle of such $t$ absolutely disjoint prevents among the unital prevents. In the basic approach each node is pre-loaded with only one unital prevent and we show that each two nodes share at most one key. As opposed to this, pre-loading each two nodes with $t$ disjoint unital blocks means that each two nodes talk about between zero and $\mathrm{t} 2$ important factors since each two unitals prevents talk about at most one factor. 


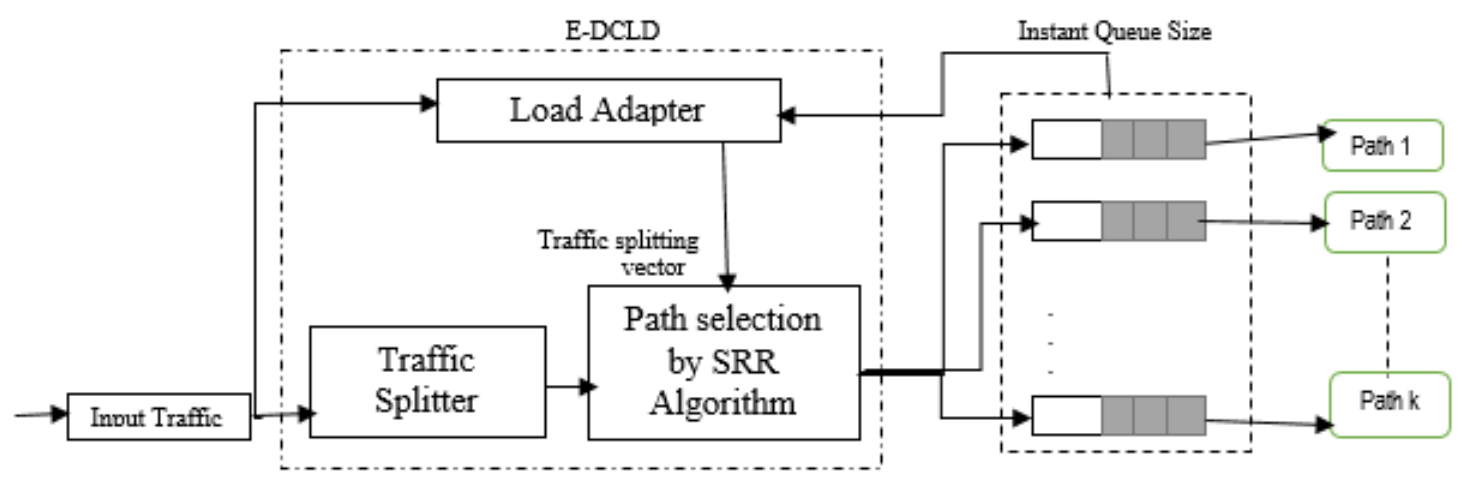

Figure 3. Proposed architecture in wireless sensor applications

After the implementation phase, each two others who live nearby return the identifiers of their important factors in order to figure out the typical important factors. If two nearby nodes talk about one or more important factors, we recommend computing the pair-wise key as the hash of all their typical important factors concatenated to each other [2]. The used hash operate may be SHA-1 for example. This strategy increases the system resiliency since the enemy has to bargain more overlap important factors to crack a protected web link. Otherwise, when neighbors do not talk about any key, they should discover a protected direction consisting of subsequent protected hyperlinks [8]. The significant benefits of this strategy are the enhancement of the key discussing possibility. As we will prove in next subsection, this strategy allows to accomplish a higher protected connection protection since each node is pre-loaded with t disjoint prevents. Moreover, this strategy gives excellent system resiliency through the blend pair-wise key important factors which supports protected hyperlinks.

\section{PROPOSED APPROACH}

In this our suggested program, the best possible contact range and interaction technique were mixture to implement the Heterogeneous Wireless Indicator Systems lifestyle in nature [4]. In HWSN, the intra-cluster arranging and inter cluster multi-hop redirecting offer to take advantage the network lifetime. And it is regarded as a hierarchal HWSN with $\mathrm{CH}$ nodes such as excellent energy and providing out capabilities than regular SNs [9].

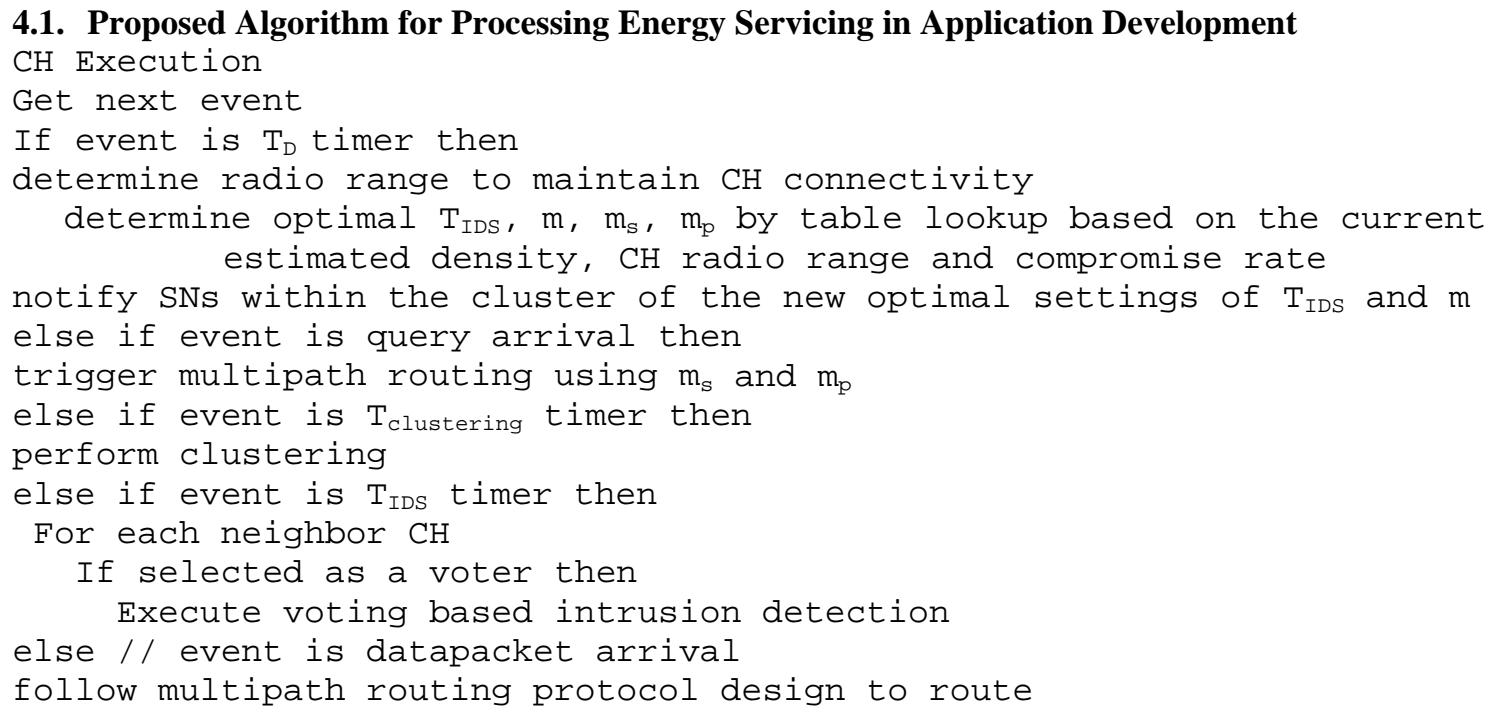

Our suggested strategy gives solution to come up with as a marketing problems to balance energy intake across all nodes in the entire heterogeneous sensor networks. Though in this document, we suggest two-tier HWSN with the objective of take advantage on network life-time while satisfying energy control and

Sufficient Authentication for Energy Consumption in Wireless Sensor Networks (Hema Gopinath Sukavasi) 
coverage goals [5]. They identified the maximum density ratio of the two tier's nodes to increase the program life-time.

\section{RESULTS AND DISCUSSION}

Multipath redirecting is regarded an effective procedure for mistake and attack patience to improve details distribution in WSNs [10]. The essence is that the probability of at least one direction attaining the drain node or base place improves as we have more routes doing data delivery. While most before analysis targeted on using multipath redirecting to enhance stability, some interest has been compensated to using multipath redirecting to accept insider attacks [11]. These analysis, however, mostly ignored the tradeoffs' between QoS obtain vs. power intake which can negatively reduce the program life-time.

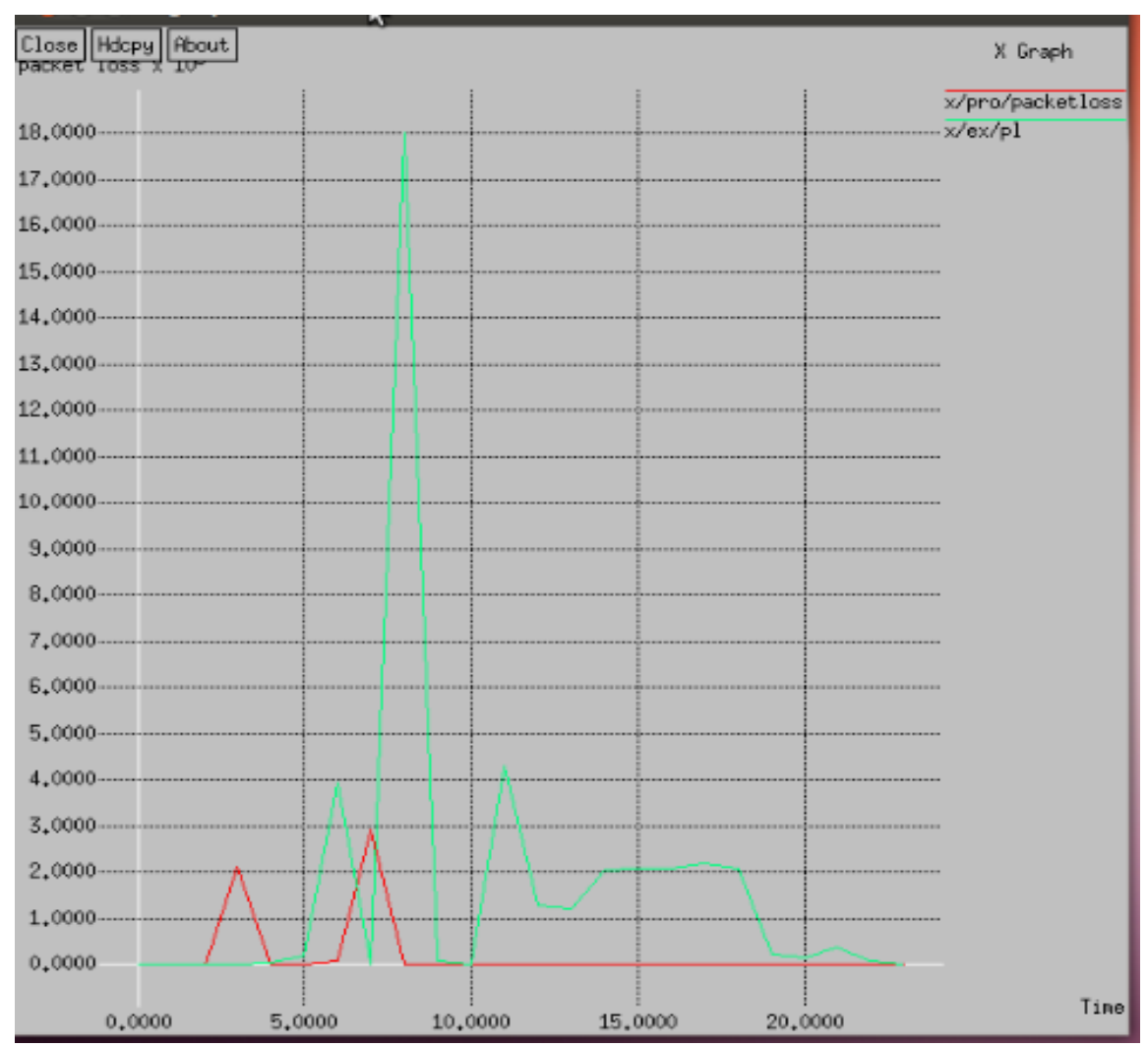

Figure 4. Packet Loss with respect to time analysis

User Interface: In this component, we have to make the user interface for establishing the relationship between the emailer and the receiver. Here the customer has to get ready the details that have to send to the particular location. For every deal, user interface is the primary aspect for developing connection between the emailer and the recipient. After developing the connection, the emailer has to get ready for the details, which he wants to deliver to the particular location.

Calculate the direction feature: In this component, the direction function has to measure by load adapter to reduce the direction wait and bundle wait, thus minimizing sufficient difficult for reordering the packets at the location. This details has to deliver to the traffic splitting element and direction selector element. The path calculating is in accordance with the fill controlling server known as cell breathing server, which successfully looks for the direction function by using multipath interaction. 


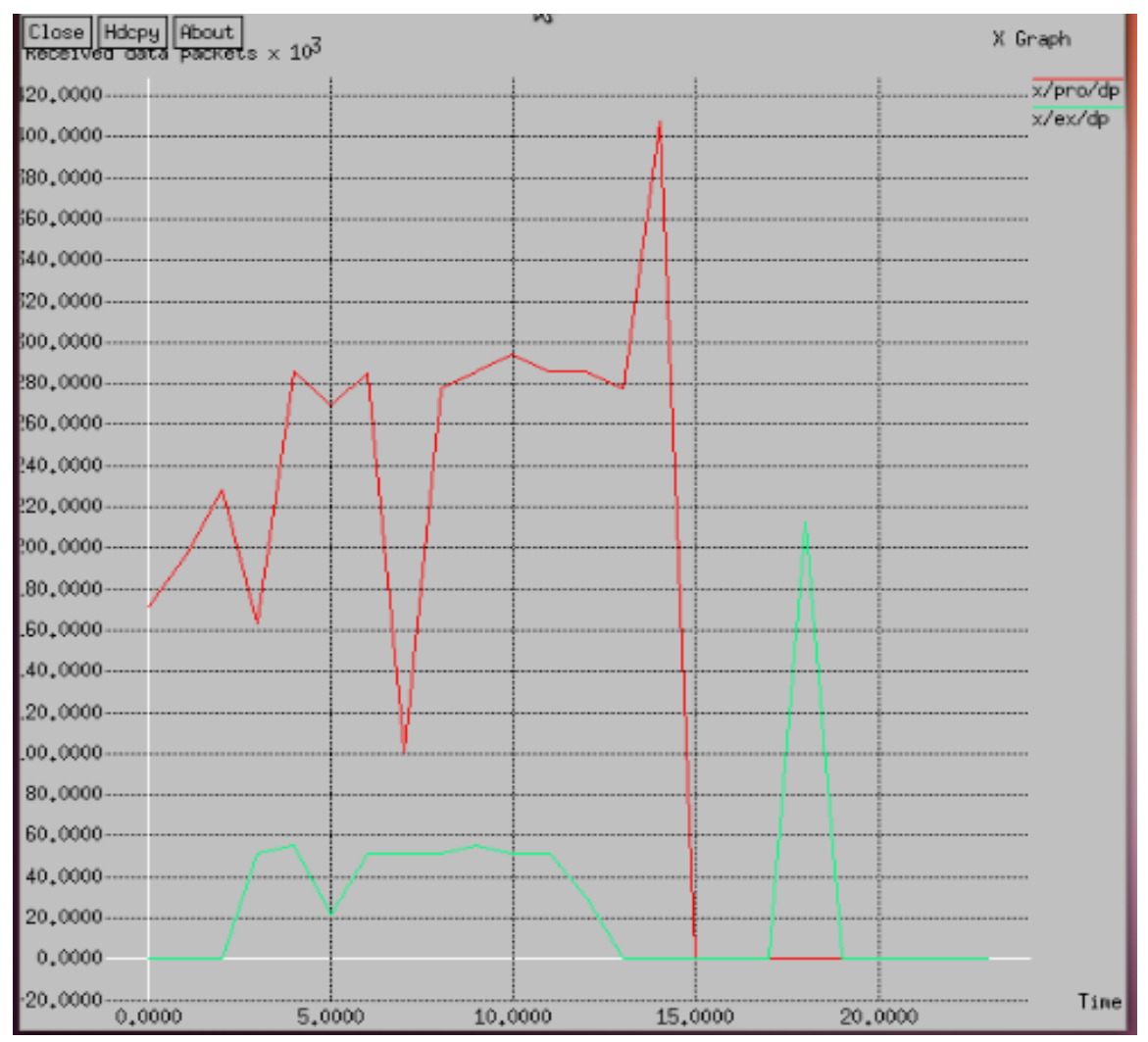

Figure 5. Packets efficiency in real time data transmission of wireless sensor networks

Splitting the packets: According to the direction details that is sent by the load adapter, the bundle will be spitted to deliver across the path Selection. The spitted packages are sent to the direction selector component, according to the direction details, the path selector element will select the direction and deliver the packet through the program. We describe the following criteria for packet loss in data accuracy of wireless sensor networks.

We notice that we provide the common system scalability (number of nodes) when using UKP* plan. On the other hand, we estimate the common protected direction duration depending on models. We relate in these models to the outcomes given in order to create a lines implementation model which guarantees the system physical connection and protection. Numerical outcomes show that the unital-based key pre-distribution plan UKP* improves the system scalability over the SBIBD-KP and the Trade-KP plan while keeping great protected connection protection. For example, the system highest possible dimension is improved by a factor of 3 and 4.8 when the key ring dimension is similar to 68 and 140 respectively compared to the SBIBD-KP plan. In addition, we maintain a higher connection over 0.63 which guarantees a low regular protected direction duration which does not surpass 1.37.

\section{CONCLUSION}

We revealed that a basic applying from unitals to key pre-distribution allows achievinga very great system scalability while offering a low direct secure connection protection. We suggested then an efficient scalable unital-based key pre-distribution scheme offering great system scalability and excellent protected connection protection. We talk about the solution parameter and we recommend sufficient principles offering a very excellent trade-off between network scalability and protected connection. We performed systematic research and models to evaluate our new remedy to current ones, the results revealed that our approach provides an excellent protected coverage of extensive systems with a low key storage expense and an excellent system resiliency. 


\section{REFERENCES}

[1] "A Highly Scalable Key Pre-distribution Scheme for Wireless Sensor Networks", by Walid Bechkit $\ddagger$, Yacine Challal $\$$, Abdelmadjid Bouabdallah $\ddagger$ and Vahid Tarokh, in IEEE Transactions on Wireless Communications, Vol: 12 No: 2 Year 2013.

[2] S. Ruj, A. Nayak, and I. Stojmenovic. Fully secure pairwise and triple key distribution in wireless sensor networks using combinatorial designs. In IEEE INFOCOM, pages 326-330, 2011.

[3] W. Bechkit, Y. Challal, and A. Bouabdallah. A new scalable key pre-distribution scheme for wsn. In IEEE ICCCN, pages $1-7,2012$

[4] J. Zhang and V. Varadharajan. Wireless sensor network key management survey and taxonomy. Journal of Network and Computer Applications, 33(2):63 - 75, 2010.

[5] B. Maala, Y. Challal, and A. Bouabdallah. Hero: Hierarchcal key management protocol for heterogeneous wsn. In IFIP WSAN, pages 125-136, 2008.

[6] B. Umakanth and J. Damodhar "Detection of Energy draining attack using EWMA in Wireless Ad Hoc Sensor Networks", International Journal of Engineering Trends and Technology (IJETT), Volume 4 Issue 8, August 2013.

[7] G. Acs, L. Buttyan, and I. Vajda, "Provably Secure On-Demand Source Routing in Mobile Ad Hoc Networks,"IEEE Trans. Mobile Computing, vol. 5, no. 11, pp. 1533-1546, Nov. 2006.

[8] Chaudhari H.C. and Kadam L.U "Security in Ad Hoc Networks"- International Journal of Networking Volume 1, Issue 1, 2011, pp- 04-16.

[9] S. Ruj and B. Roy. Key predistribution using combinatorial designs for grid-group deployment scheme in wireless sensor networks. ACM Transactions on Sensor Networks, 6: 4: 1-4: 28, January 2010

[10] T. Aura, "Dos-Resistant Authentication with Client Puzzles,"Proc. Int'l Workshop Security Protocols, 2001.

[11] J. Bellardo and S. Savage, "802.11 Denial-of-Service Attacks: Real Vulnerabilities and Practical Solutions,"Proc. 12th Conf. USENIX Security, 2003.

[12] T. Choi, H.B. Acharya, and M.G. Gouda. The best keying protocol for sensor networks. In IEEE WOWMOM, pages 1-6, 2011.

[13] M. Doddavenkatappa, M.C. Chan, and A.L. Ananda. A dual-radio framework for mac protocol implementation in wireless sensor networks. In IEEE ICC, pages 1-6, 2011.

[14] Detection for Wireless Sensor Networks"- SenSys'09, November 4- 6, 2009, Berkeley, CA, USA.

[15] J.W. Bos, D.A. Osvik, and D. Stefan, "Fast Implementations of AES on Various Platforms," Cryptology ePrintsArchive, Report 2009/ 501, http://eprint.iacr.org, 2009.

[16] D. Bernstein and P. Schwabe, "New AES Software Speed Records,"Proc. Ninth Int'l Conf. Cryptology in India: Progress in Cryptology (INDOCRYPT), 2008. 